\title{
COLLOIDAL SUSPENSION OF GRAPHITE
}

\section{BY HENRY L. DOYLE}

Experiments were made at first to determine whether substances, which are adsorbed by carbon and graphite, have a marked peptonizing action on them. Boneblack and Acheson graphite were used and were ground in an agate mortar. In the earlier experiments they were ground dry, but later they were ground wet, being ground for one hour in the solutions in which they were to be suspended.

Since Spring ${ }^{1}$ had found that soap solutions would wash rouge or boneblack through filter paper, this seemed to offer a promising line of attack. Finely ground boneblack was placed on a filter and washed with distilled water until the wash water ran through colorless. The residue on the filter was then washed with a 5 percent sodium oleate solution. Carbon was carried through the filter. A similar experiment was then made with graphite and a similar result obtained. Negative results were obtained with boneblack, using 0.5 percent and 5.0 percent solutions of sodium chloride, cupric nitrate, caustic soda, acetic acid, hydrochloric acid, and gelatine. ${ }^{2}$ Negative results were obtained with saturated and halfsaturated solutions of mercuric chloride; and also with chlorine water. The 5 percent sodium oleate solution not only carried the carbon through the filter but held it pretty well in suspension over night. In fact, the carbon had not all settled at the end of two days.

All of these substances are adsorbed readily by some forms of carbon and yet only one of them, soap, carried the boneblack through the filter paper. Since there was a possibility of a disturbing effect due to the filter paper, it was decided to change to suspensions in stand-glasses and to note the relative rates of settling.

A considerable amount of boneblack was ground up and

\footnotetext{
'Zeit. Kolloidchemie, 4, I6I (Ig09); 6, II, 109, 164 (IgIO).
}

2 Ammonia was added to the 5 percent gelatine. 
the same amount, so nearly as possible, was taken for each experiment. The experiments were made in stand-glasses holding about 500 cc. Since there might be an optimum concentration, experiments were made with 0.5 , I.o and 5.0 percent solutions of sodium chloride, acetic acid, chlorine water, caustic soda, hydrochloric acid, cupric nitrate, gelatine, sugar, and soap. Only with the last three substances was there any noticeable suspension. The most dilute soap solution kept up a little boneblack, the I percent solution more, while the 5 percent solution was quite efficient. The most concentrated sugar solution did fairly well; but the results were negative with the 0.5 percent solution. Only the most concentrated gelatine solution had any protecting action and that one not much. The 5 percent gelatine could not be kept fluid enough at ordinary temperatures. Consequently, the experiment was made at about $50^{\circ}$ in a water bath, using a roo ce stand-glass. The largest particles are formed in the acetic acid, hydrochloric acid, and chlorine solutions, while fairly large particles are formed in the cupric nitrate solutions. With hydrochloric acid there was a visible evolution of gas, presumably from calcium carbonate in the boneblack.

Since sodium oleate and gelatine both keep up the carbon, and since both lower the surface tension of water, it seemed worth while to consider whether solutions of the two having the same surface tension have approximately the same effect in keeping boneblack in suspension. The weight per drop from a given orifice was found to be 0.042 gram for water, 0.038 gram for a 2 percent gelatine solution, and 0.016 gram for a 2 percent sodium oleate solution. Since the lowering of the surface tension of water is much greater for a given weight of soap than for an equal weight of gelatine, it was necessary to keep down to a low soap concentration. It was hoped that a gelatine solution might be made having as low a surface tension as a $\mathrm{I}$ percent sodium oleate solution. This proved to be impossible, since a so percent gelatine solution gives a much higher drop weight at $60^{\circ}$ than a $\mathrm{I}$ percent soap solution at ordinary temperatures. An attempt was made to 
keep the gelatine solution fluid at lower temperatures and a solution was therefore made up containing ro percent gelatine and 5 percent ammonium sulphocyanate. This was so viscous at ordinary temperatures that experiments with it seemed useless.

It seemed desirable to try some experiments with a dissolved substance which was not an electrolyte and which was readily adsorbed by carbon. Iodine was apparently the most satisfactory substance for this purpose; but its sparing solubility in water made another solvent necessary. Benzene was the first one tried. Benzene solutions were made up containing $\mathrm{I}-5$ percent iodine. Boneblack was ground with some of the benzene solution in an agate mortar for an hour and was then poured into the rest of the benzene solution in a Ioo cc glass cylinder. The time was determined for the boneblack to settle practically completely. Check experiments were run with benzene, containing no iodine. No difference could be detected due to the iodine. The experiments were repeated, allowing the mixtures to stand for a definite time, and then filtering. The residues were washed with benzene until all traces of iodine were removed. The two sets of residues on the filters were identical so far as the eye could tell.

A corresponding set of runs was also made with graphite instead of boneblack. The same results were obtained. Since graphite did not stay up well in benzene and since it did not grind easily in this liquid, a change was made to absolute alcohol

At the end of a given time the bulk of the liquid in a cylinder was decanted and enough caustic potash solution added to decolorize the iodine. Of course the same amount was added in the check test where the alcohol contained no iodine. With boneblack, one run gave more carbon suspended in the iodine solution, one the same amount, and one less. The experimental error seems to be rather large; but there is no proof of any increased suspension due to the iodine. Negative results were also obtained with graphite. 
Since the adsorbing power of boneblack varies very much with the previous history of the sample ${ }^{1}$ and since it was not certain whether the graphite adsorbed any appreciable amount of iodine, some experiments were made in regard to this. Saturated solutions of iodine in water were made up and then filtered so as to remove all particles of undissolved iodine. The filtered solutions were placed in $500 \mathrm{cc}$ glass-stoppered bottles. One was shaken with boneblack and the other with Acheson graphite. After settling, the bottle containing the boneblack was much less colored than the one containing graphite. After several shakings, the liquid was completely decolorized in the bottle containing boneblack while the liquid in contact with graphite was changed but slightly. This seemed to show that iodine was only slightly adsorbed by graphite and that the adsorption by boneblack was very much greater. The bottles were put away for two weeks however and at the end of that time the liquid in contact with graphite was also decolorized. This looked as if the difference were more a matter of rate of adsorption than of total adsorption.

Since the rate of adsorption of iodine by graphite is evidently low, it seemed wise to repeat the experiments on suspension by shaking a solution of iodine in alcohol for a long time with graphite, making a parallel run with a similar bottle containing graphite and alcohol only. To one bottle was added $60 \mathrm{cc}$ absolute alcohol, I gram graphite and 6 grams iodine, while the other bottle contained $60 \mathrm{cc}$ absolute alcohol and I gram graphite but no iodine. The two bottles were shaken for several days a few hours a day and then allowed to stand. Equal portions of the liquids were decanted into glass cylinders and equal quantities of a dilute caustic potash solution added to each. The amount added was sufficient to decolorize the iodine solution. Owing to the alcohol iodoform was formed. Each solution was filtered through a tared filter and the iodoform was dissolved with ether. The ether

${ }^{1}$ Pelet-Jolivet: Die Theoric des Färbeprozessess, 58 (IgI I). 
apparently extracted some iodine from the graphite as well. The filters were dried and weighed. There was slightly more graphite from the bottle containing iodine than from the other. So far as this one set of experiments shows anything, the iodine does keep the graphite up a little. The experiment was not repeated because the real conclusion to be drawn from it seemed to be that iodine keeps graphite in suspension very slightly, if at all.

Since boneblack varies very much in composition, a sample was ignited. It gave 83.5 percent of ash, presumably calcium phosphate. It is quite possible that different results would have been obtained with a boneblack from which the calcium phosphate had been removed; but it seemed wiser at first to work with material similar to that used in most experiments on adsorption. Incidentally an ash determination was made on a sample of natural graphite marked C. P. It showed 43 percent of ash. This material was not used in any of the experiments.

It was now decided to try the effect of protecting colloids. As a preliminary, a sample of aquadag was analyzed. The paste was dried on an air bath at about $100^{\circ}-125^{\circ}$. When nearly dry, the hard cake was ground in an agate mortar, dried and ground again. In a gram sample, determinations were made of volatile matter 9 percent, and ash 8 percent. The ash was dissolved in sulphuric acid, filtered and precipitated with ammonia. The ferric oxide was dried and weighed. The copper was determined electrolytically. The dried sample of aquadag contained 3.2 percent $\mathrm{Fe}_{2} \mathrm{O}_{3}$ and $\mathrm{I} .2$ percent $\mathrm{Cu}$.

It did not seem probable that aquadag would contain so much iron unless this were beneficial in some way. To test this, a fairly dilute solution of ferric acetate was boiled for a few minutes so as to hydrolyze it. One gram of finely ground graphite was added to $60 \mathrm{cc}$ of this solution and I gram to $60 \mathrm{cc}$ water. The mixtures were placed in roo ce bottles and were shaken for an hour and a half with a power shaker. The bottles were allowed to stand for 2 hours when equal amounts of the supernatant liquid were poured off, sulphuric 
acid being added to dissolve the colloidal ferric oxide. There was more graphite suspended in the liquid from the bottle containing iron. To the residues in the bottles, water was added, and the bottles shaken as before. As before, more graphite was in suspension in the liquid from the bottle to which iron had been added. Water was added again to the residues with the same result. When these last solutions were filtered, a distinct difference could be seen in the character of the graphite. In the one containing iron, the particles were fine and covered the entire surface of the filter, whereas the particles from the water were agglomerated and formed little heaps. These experiments show that a hydrolyzed ferric acetate solution keeps finely-divided graphite in suspension to a certain extent. It would have been rather gratifying to have tried these experiments and the later ones with the same graphite which is used in making aquadag and oildag; but the Acheson company refused to sell any.

Experiments were now made with mixtures of iron salts with organic substances, beginning with gelatine. Half a gram of graphite powder, half a gram of gelatine and about o. I gram ferric chloride were placed in a bottle together with $60 \mathrm{cc}$ water and a lot of glass beads. Another bottle was equipped in the same way except that the ferric chloride was omitted. Both bottles were shaken for 4 hours in a power shaker. On standing, no difference could be detected between the bottles. The contents of the bottles were then diluted to $500 \mathrm{cc}$ and allowed to stand. If anything, the suspension was less satisfactory in the bottle containing the ferric chloride. Similar experiments using less ferric chloride, were no more satisfactory. Substituting 0.5 gram tannic acid for the gelatine and shaking for ro hours, did not improve matters, nor did cutting down the tannic acid to o.I gram.

The experiment with tannic acid was repeated, adding ammonia until it could be detected by the smell. The bottle was shaken and opened from time to time to find out whether the solution was still ammoniacal. If not, more ammonia was added. The result was apparently a beautiful black 
suspension which remained black. Unfortunately, most of this was due to the formation of an iron ink, which ought to have been foreseen. Similar experiments with catechu gave no satisfactory results. It seemed possible that the method was faulty, so experiments were made to find out a good method for getting catechu into suspension. In one case the catechu was ground and heated with water, after which ammonia was added; in the other case the ammonia was added to the catechu before the heating took place. The second method gave better suspensions of catechu. Qualitative experiments were then made as to the action of an ammoniacal suspension of catechu on graphite. To one of the bottles fine iron filings were added. Both bottles were shaken in the power shaker; the solutions were diluted and stirred by bubbling air through them. After standing over night, equal amounts were taken from each cylinder and precipitated with concentrated hydrochloric acid. The precipitates were separated by filtration. There was a larger residue from the bottle containing iron. The experiment was repeated several times, always with the same result.

A few quantitative runs were made using a suspension containing $25 \mathrm{cc}$ water, 0.5 catechu and ro cc ammonia as deflocculating agent. In the first run 5 grams graphite and 0.5 gram $\mathrm{Fe}$ (5 grams graphite and no iron in the check experiment) were ground for 2 hours in a McKenna grinder with Io cc of the deflocculating agent, a little water being added when necessary to keep the mass of the right consistency. The ground mass was diluted to rooo $\mathrm{cc}$ and Ioo $\mathrm{cc}$ samples pipetted off after standing. After precipitation with concentrated hydrochloric acid, the precipitates were collected on tared filters and weighed. In some cases still greater dilutions were taken. The following results were obtained:

$\begin{array}{lll} & \begin{array}{c}\text { No iron } \\ \text { Gram }\end{array} & \begin{array}{c}\text { With iron } \\ \text { Gram }\end{array} \\ & & 0.0812 \\ \text { Weight of precipitate } & 0.0350 & 0.0275 \\ \text { Weight of precipitate } & 0.0110 & 0.0185 \\ \text { Weight of precipitate } & 0.0092 & \end{array}$


The presence of iron causes at least twice as much graphite to be kept in suspension by an ammoniacal catechu suspension as would be the case without iron. The character of the suspension is better as shown by filter-paper tests. The character of a deposit can be judged by the edge of the circle where the drop is placed. There should be no saw-tooth effect; the color should go through the paper and also extend out as far as the water does, or nearly as far.

No experiments have been made with copper salts; but it is interesting to note that Pickering ${ }^{1}$ obtained good emulsions of kerosene in water with basic ferric sulphate or cupric sulphate as emulsifying agent.

At the suggestion of Mr. Briggs I tried a solution of caseine in sodium phosphate as a means of keeping graphite in suspension. A very good suspension was obtained which seemed to stay up indefinitely and which was not very sensitive to acids.

Experiments were next made to determine, if possible, under what conditions graphite could be suspended in machine oil. Catechu could not be used because it did not dissolve in machine oil and seemed to undergo a decomposition when heated in oil. When ground with graphite and oil, catechu gave no suspensions, or rather the suspensions settled at once. It was, therefore, necessary to find some other colloid, and it seemed as though the best thing would be a gum or resin which would dissolve in hot oil.

The first substance to be tried was resin; but no satisfactory results were obtained with this. Melted resin was added to hot or boiling oil; but the graphite did not stay in suspension unless the mixture was very thick. The procedure was to heat about I gram of resin until it was volatilizing rapidly, to add $75 \mathrm{cc}$ hot oil, and then to boil. Of the resulting mixture, amounts varying from one drop to $5 \mathrm{cc}$ were added to enough oil to make $15 \mathrm{cc}$ and the whole ground with 2 grams of graphite for 30 minutes in a McKenna grinder.

${ }^{1}$ Jour. Chem. Soc., 9I, 2001 (1907). 
In no case would a dilute solution of resin keep the graphite in suspension.

Shellac did not dissolve in the oil and asphaltum did not keep the graphite up until the concentration was high enough to make the mass sticky.

It next occurred to me that perhaps ferric oxide might work well. Graphite was therefore ground first with moist precipitated ferric hydroxide and afterwards with oil. A suspension of graphite was obtained in this way, but it was not very satisfactory. I varied the relative amounts of ferric hydroxide and water from an almost dry paste to a liquid, and the absolute amounts from a drop to a cubic centimeter. The best suspension was obtained with small amounts both of water and of ferric hydroxide. The method seems to have possibilities if the grinding with the oil could be carried on at a temperature at which the water would evaporate until the protecting action of the ferric hydroxide was a maximum.

Since soap had proved serviceable when making suspensions of graphite in water, it seems worth while to try something similar for suspensions in oil. In view of the fact that paint-makers find that the addition of a little water enables them to keep the paint up better in the oil, the addition of water was also to be considered. In the first runs sodium oleate was used, the soap and water being ground with the graphite for ro minutes. The oil was then added, a little at a time, the grinding being kept up for 20 minutes longer. The following results were obtained with 2 grams graphite, o.I gram sodium oleate, I5 cc oil, and varying amounts of water.

\begin{tabular}{l|l} 
Water & \multicolumn{1}{c}{ Suspension } \\
\hline & \\
$0.5 \mathrm{cc}$ & Stiff emulsion cracking soon \\
$0.3 \mathrm{cc}$ & Stiff emulsion cracking soon \\
$0.2 \mathrm{cc}$ & Stayed up somewhat longer \\
$0.1 \mathrm{cc}$ & Stayed up thirty minutes \\
2 drops & Fair suspension \\
I drop & Fair suspension \\
0 & Good suspension for I2 hours \\
& Not so good as preceding
\end{tabular}


Magnesium soap was tried next and was made by heating sodium oleate with a magnesium sulphate solution, the magnesium oleate separating. As in the case of the sodium oleate, the best results were obtained with only one drop of water. The suspension with magnesium oleate seemed to be better than the one with sodium oleate.

Since iron oxide proved beneficial in keeping graphite suspended in water, it seemed probable that an iron soap might be an excellent substance with which to make a suspension of graphite in oil. I first tried heating ferric acetate with sodium oleate. I got a small amount of a substance which I thought might be an iron soap; but it was not a success as a protecting colloid. I next tried to prepare an iron soap by heating linseed oil with ferric oxide. After several attempts in which I did not get anything much like a soap, I finally got a viscous liquid which may have been a soap; but which contained a lot of decomposition products and which did not work well with oil and graphite. I went back to the first method; but substituted ferric chloride for the acetate. On heating ferric chloride solution with sodium oleate, I obtained a gummy mass which was insoluble in water and which separated readily. With this gummy material a series of experiments was made precisely similar to those with sodium oleate and magnesium oleate. So long as any water was added, I found it impossible to get any satisfactory results, because the soap remained in viscous particles and settled rapidly.

On the other hand, graphite with this iron soap and oil but no water gave better suspensions than I have been able to get in any other way. The amount of soap could vary between 0.1 gram and I.O gram per $20 \mathrm{cc}$ and still give good suspensions. In one case, only $1 / 8$ inch of oil could be seen above the suspension after standing for two weeks.

While working with the iron soap, I also tried heating paraffin for about Ioo hours. A dark, gummy residue was obtained which gave fairly good suspensions when ground 
with graphite and oil. The results were not as satisfactory as those obtained with the iron soap.

The general results of this paper are:

I. Experiments have been made on the suspension in water of boneblack having 83.5 percent ash, and on the suspension in water and in oil of finely-ground Acheson graphite.

2. Sodium oleate will hold boneblack or graphite in aqueous suspension; concentrated solutions of sugar or gelatine have a slight effect.

3. Although sodium chloride, cupric nitrate, caustic soda, chlorine, acetic acid, and hydrochloric acid are adsorbed readily by boneblack, no one of these substances had any appreciable effect in keeping boneblack or graphite suspended in water.

4. Iodine was tried with boneblack and with graphite in benzene and in absolute alcohol; but there was no proof that it was beneficial.

5. The boneblack decolorized a saturated solution of iodine in water fairly quickly; the graphite decolorized the solution also, but very slowly.

6. A sample of dried aquadag was found to contain 3.2 percent $\mathrm{Fe}_{2} \mathrm{O}_{3}$ and $\mathrm{I} .2$ percent $\mathrm{Cu}$, which formed part of the 8 percent ash.

7. A hydrolyzed ferric acetate solution kept boneblack and graphite in suspension in water.

8. Adding ferric chloride to gelatine, tannic acid, or catechu solutions did not have any beneficial effect.

9. Better suspensions of catechu are obtained by adding ammonia before heating instead of after heating.

Io. When graphite was ground with ammoniacal catechu suspension and the mixture then shaken with iron filings and air, much more graphite was held in suspension than by catechu alone.

I I. A good suspension of graphite in water can be obtained by using a solution of caseine in sodium phosphate.

I2. No satisfactory suspension of graphite in oil could be obtained by means of resin or shellac. 
13. A fair suspension of graphite in oil can be obtained by means of ferric hydroxide and water, using very little of both.

I 4. Good suspensions of graphite in oil can be obtained by means of sodium oleate or magnesium oleate and a trace of water. A good proportion is $15 \mathrm{cc}$ oil, 0.1 gram sodium or magnesium oleate, and one drop of water.

15. An iron soap was prepared by heating ferric chloride solution with sodium oleate. Excellent suspensions of graphite in oil were obtained by means of this iron soap. No water should be added.

I6. A fair suspension of graphite in oil was obtained by means of a gummy product prepared by heating paraffin in the air.

This investigation was suggested by Professor Bancroft and has been carried on under his supervision. 